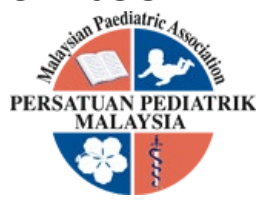

\title{
INTRAOSSEOUS MYOFIBROMA OF THE MANDIBLE IN A 7-YEAR-OLD PATIENT: A CASE REPORT
}

\author{
Meshala Bala Sundram ', Navasheilla Retna Retnasingam ${ }^{1}$, Bahruddin Saripudin', \\ Zuraiza Mohamad Zaini ${ }^{2}$
}

\begin{abstract}
Myofibroma is a benign fibrous tumour that occurs predominantly in the head and neck region followed by the trunk and extremities. However, cases occurring in the oral cavity are rare, presenting with a variable clinical appearances and wide differential diagnosis. We reported a case of a 7-year-old girl who was referred to the Department of Paediatric Dentistry, Tengku Ampuan Rahimah Hospital with a progressively enlarging painless swelling on the left posterior region of the mandible over the past 3 months. The swelling was associated with ulceration and displaced lower left first permanent molar. Radiographic investigations reported well-defined radiolucency at molar area, alveolar expansion and bone resorption of the left posterior alveolar ridge of the mandible. Complete surgical excision of the lesion was performed under general anaesthesia. Histopathological examination revealed proliferation of spindle shaped cells with biphasic growth pattern. Immunohistochemical staining showed strong positivity with vimentin and smooth muscle actin whilst negative for desmin, S100 and CD34 establishing the diagnosis of myofibroma. Although rare, myofibroma should be considered as a differential diagnosis for solitary firm swelling in the oral cavity. Histopathological examination together with immunohistochemical analysis is essential for an accurate diagnosis.
\end{abstract}

Keywords: Myofibroma, Spindle cell, Biphasic growth

\section{Introduction}

Myofibroma is a solitary benign tumour composed of myeloid cells that affects soft tissue, bone or internal organs in both children and adults [1]. Myofibroma occurs predominantly in the head and neck region followed by the trunk and extremities [2]. Cases occurring in the oral cavity are rare with the mandible being the most common site followed by the tongue, buccal mucosa and lips [2,3]. The tumour may present as a solitary or multicentric lesion known as myofibroma and myofibromatosis respectively [4]. Variability of clinical appearance combined with its rare nature leads to a wide differential diagnosis of the tumour. Misinterpretation of the lesion can lead to the wrong diagnosis and subsequently incorrect management of the lesion.
Received: 28 September 2020; Accepted revised

manuscript: 04 December 2020

Published online: 25 January 2021

\section{Case report}

A 7-year-old girl was referred to the Department of Paediatric Dentistry, Tengku Ampuan Rahimah Hospital with a progressively enlarging painless swelling on the left posterior region of the lower dentition over the past 3 months. The father denied any history of trauma, fever, weight loss and other constitutional symptoms for the patient. The past medical, dental and family history was insignificant. Extraorally, no swelling

\footnotetext{
'Department of Paediatric Dentistry, Hospital Tengku Ampuan Rahimah Klang, Malaysia

${ }^{2}$ Department of Oral and Maxillofacial Clinical Sciences, Faculty of Dentistry, University Malaya, Malaysia

Corresponding Author:

Dr. Meshala Bala Sundram, Department of Paediatric Dentistry, Hospital Tengku Ampuan Rahimah, Jalan Langat, 41200 Klang, Malaysia.

Tel: +60333756285 ; Fax: +60333734952

Email: meshala_b@yahoo.com
} 
was seen, skin was normal and there was no submandibular lymphadenopathy. Intraorally, the patient had a mixed dentition of primary and permanent teeth. A localised swelling was noted at the lower left alveolar ridge. A lesion measuring about $3.0 \times 2.5 \mathrm{~cm}$ was noted on the lingual aspect of the lower left second deciduous molar (tooth 75) extending distally to the lower left second permanent molar (tooth 37 ) region. It was noted to be erythematous with surface ulceration and buccolingual expansion of the alveolar ridge. The lesion appeared pedunculated upon palpation. The lesion was firm in consistency, not tender, associated with buccolingual expansion of alveolar ridge along with mobile and buccally displaced lower left first permanent molar (tooth 36). There was no obliteration of the lower left buccal sulcus. (Figure 1).

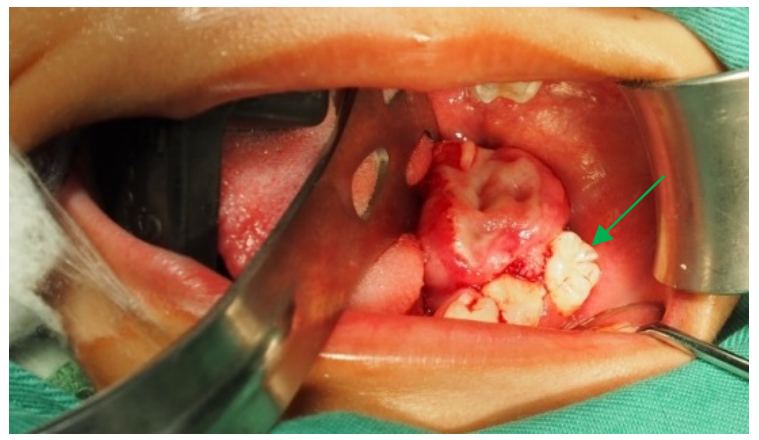

Figure 1. Intraoral photograph showing lesion on the lower left posterior region of mandible displacing tooth 36 (arrow) and 75.

Blood tests showed normal full blood counts and C-reactive protein. Aspiration of the lesion with syringe was carried out at the first visit. However, no fluid nor blood was yielded. This ruled out the possibility of the lesion being a cystic, or a blood vessel anomaly such as hemangioma. Orthopantomogram (OPG) and Cone Beam Computed Tomography (CBCT) was carried out as part of the investigation. OPG showed well defined unilocular radiolucent lesion extending from tooth 75 to 36 . No root resorption was noted in relation to tooth 36 (Figure 2). CBCT revealed buccolingual expansion with thin cortical plate and bone resorption on lower left posterior alveolar ridge (Figure 3). Electric pulp test and cold test were performed but did not elicit sensory response from tooth 36 and tooth 75 . Investigation findings led to a few provisional diagnosis such as peripheral giant cell granuloma, unicystic ameloblastoma, odontogenic keratocyst and ameloblastic fibroma.

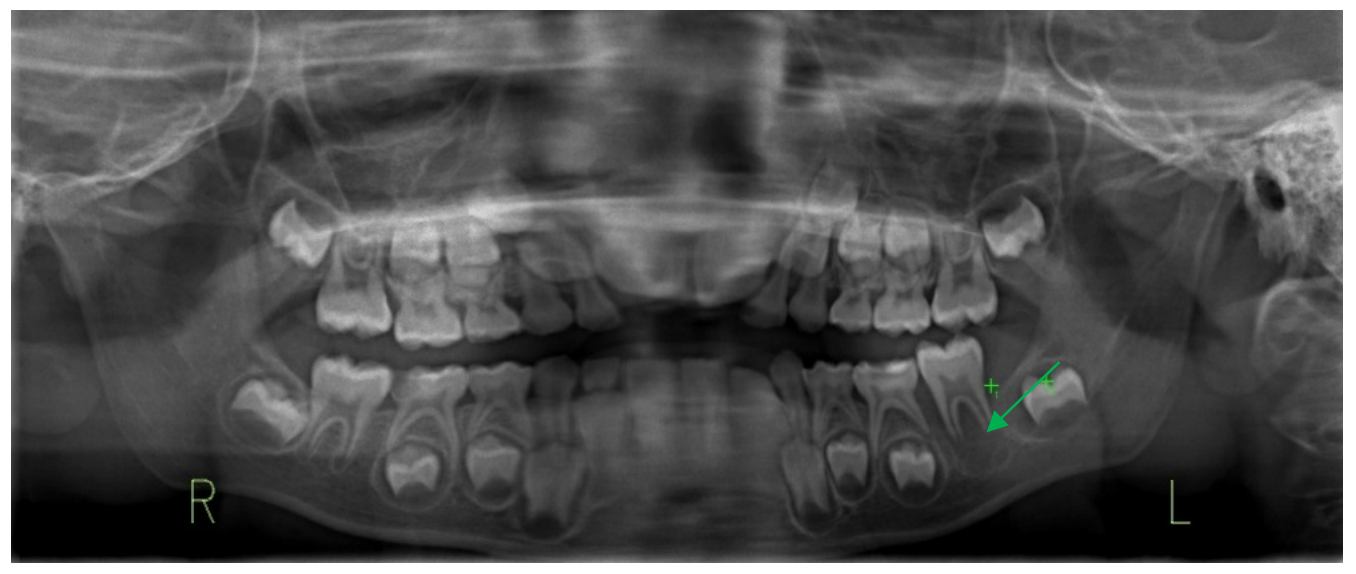

Figure 2. Orthopantomogram showing bone loss in relation to 36 region 


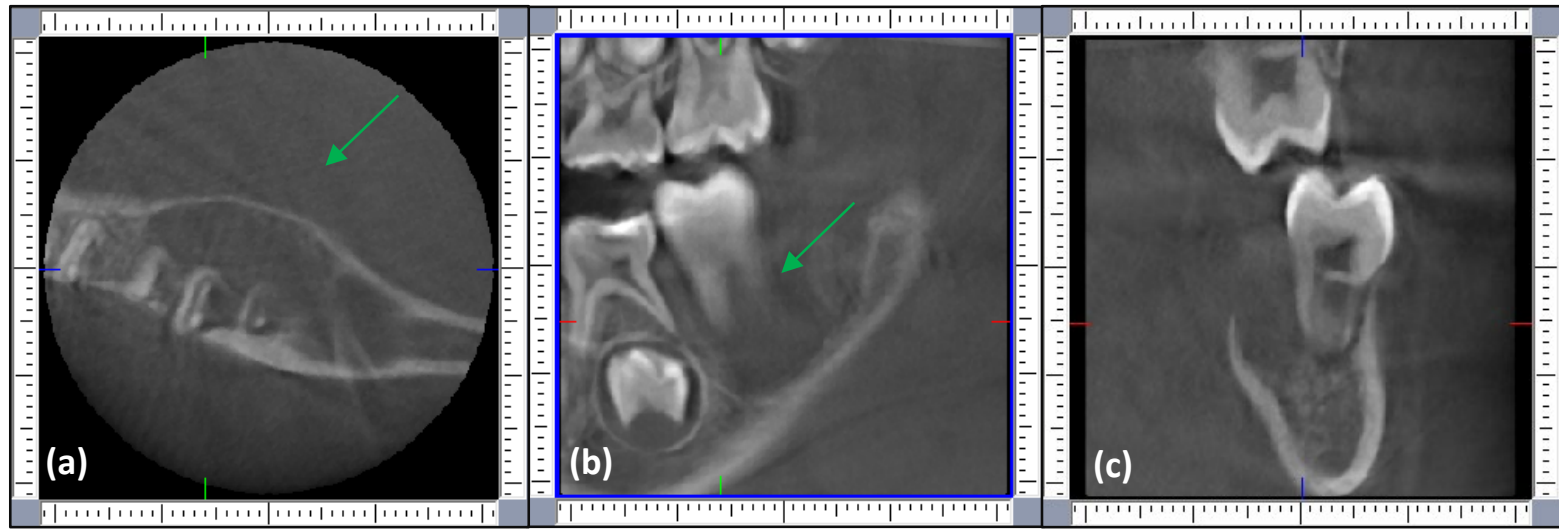

Figure 3. Cone beam computed tomography showing: (a) buccolingual expansion of alveolar ridge with resorption of lingual plate of mandible (b) radiolucency surrounding tooth 36 (c) bone resorption of the lingual of the lesion.

Treatment options were discussed and the parent consented to the decision to carry out excisional biopsy of the lesion with extraction of non-vital tooth 36 and 75 under general anaesthesia. Excisional biopsy of the lesion with a $1 \mathrm{~mm}$ surgical margin and extraction of tooth 36 and 75 was done under general anaesthesia (Figure 4). Surgical site was dressed with bismuth iodine paraffine paste (BIPP) soaked gauze enclosed within a soft splint (Figure 5).

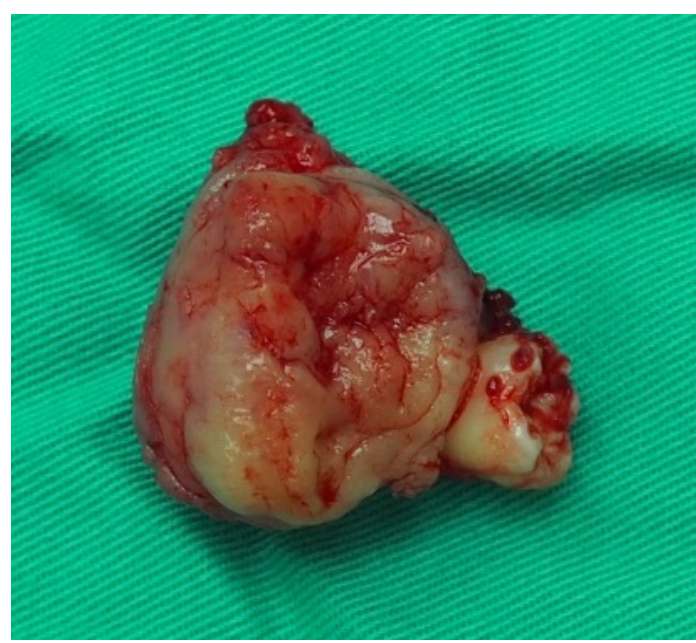

Figure 4. The excised lesion measuring $3.0 \times 2.5 \times 1.8 \mathrm{~cm}$.

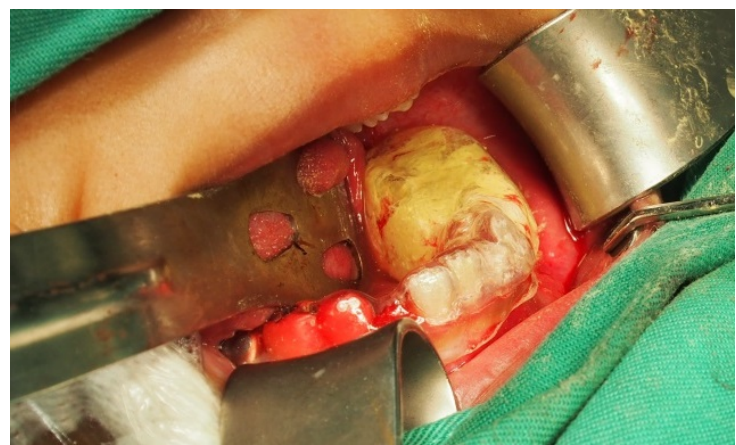

Figure 5. Post-operative photograph showing soft splint with BIPP soaked gauze as dressing to close the surgical site. 
Microscopic examination of haematoxylin and eosin (H\&E) revealed a biphasic mesenchymal tumour that was covered by an ulcerated epithelial surface (Figure 6a). The tumour cells were composed of an admixture of spindle shaped cells with elongated cigar-shaped vesicular nuclei and round to polygonal cells with scanty cytoplasm (Figure 6b). Whorl of tumour cells and intimate association with hemangiopericytoma-like vascular spaces were seen (Figure 6b). Mitosis was absent indicating the benign nature of lesion. Based on the cellular morphology, immunohistochemistry was carried out which confirmed the tumour cells lineages of mesenchymal and smooth muscles with strong positivity to vimentin and smooth muscle actin (SMA) respectively (Figure $6 c$ and $6 d$ ). Incomplete smooth muscle differentiation was established by positive staining to smooth muscle actin (SMA) whilst negative to desmin (Figure 6d). Negative immunoreactivities against S100 and CD34 further distinguished from tumours of neural and endothelial origins respectively. (a)

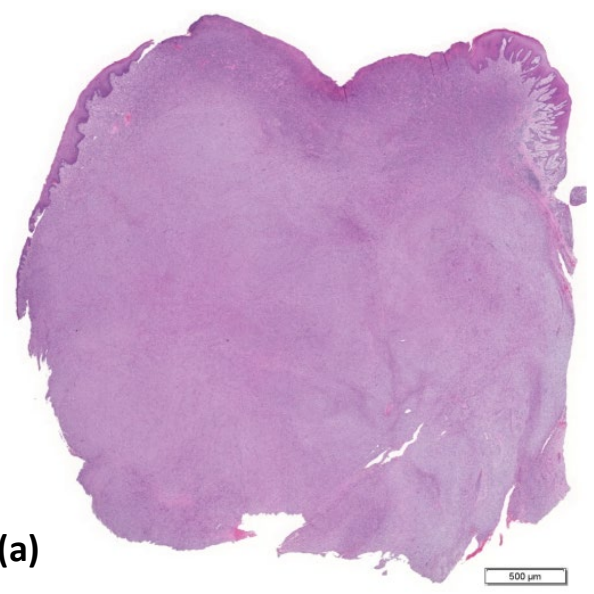

(c)

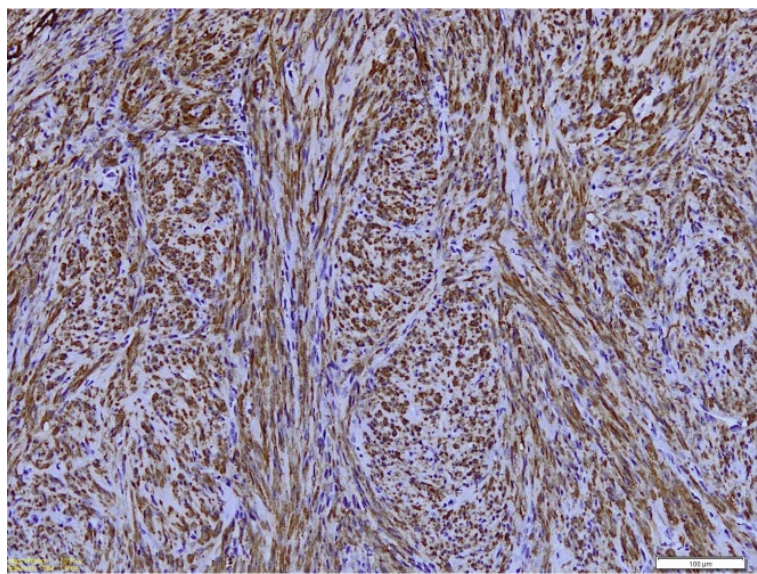

(b)
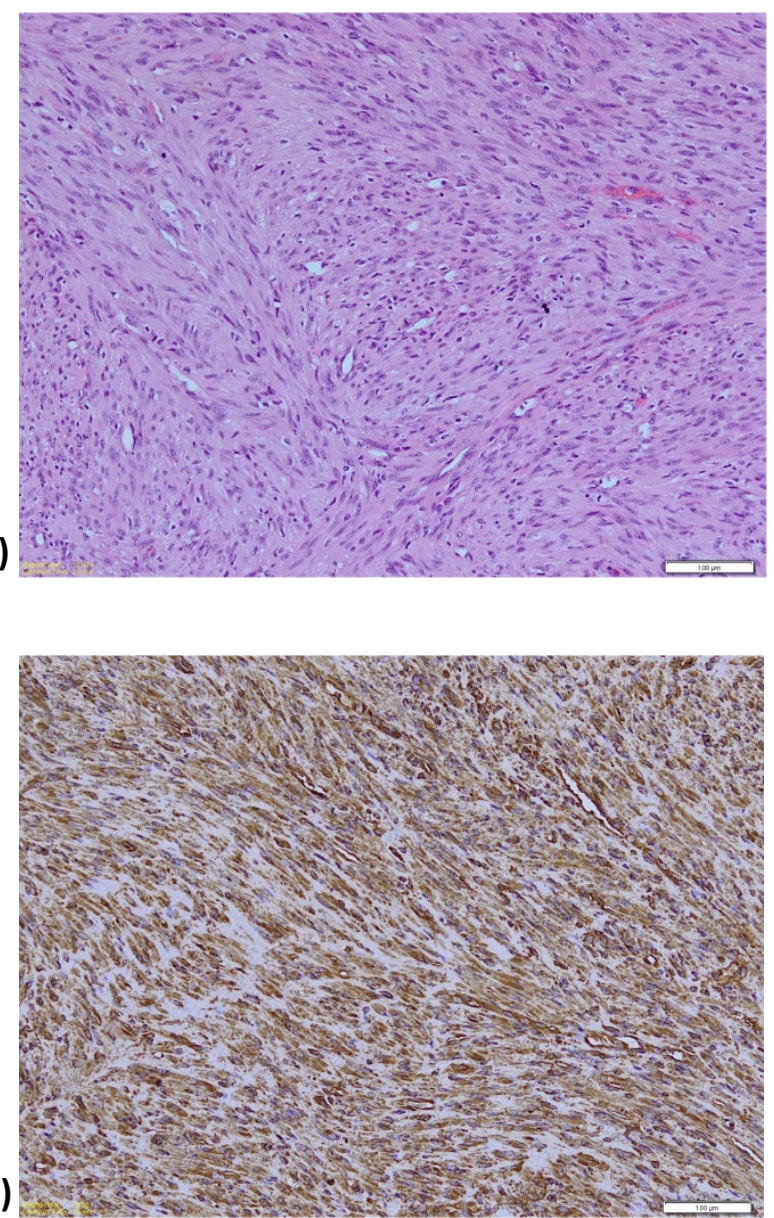

Figure 6. Histopathological characteristics demonstrating (a) biphasic pattern of distinct hyper and hypocellular zones seen under low magnification (H\&E stain, 40x magnification); (b) fascicles of myoid-like spindle cells alternating with closely packed round to polygonal cells surrounding numerous slit-like vascular spaces (H\&E stain, 200x magnification); Tumour cells show diffuse and strong immunoreactivities for (c) vimentin and (d) SMA (Envision HRP/DAB technique, 400x magnification) 

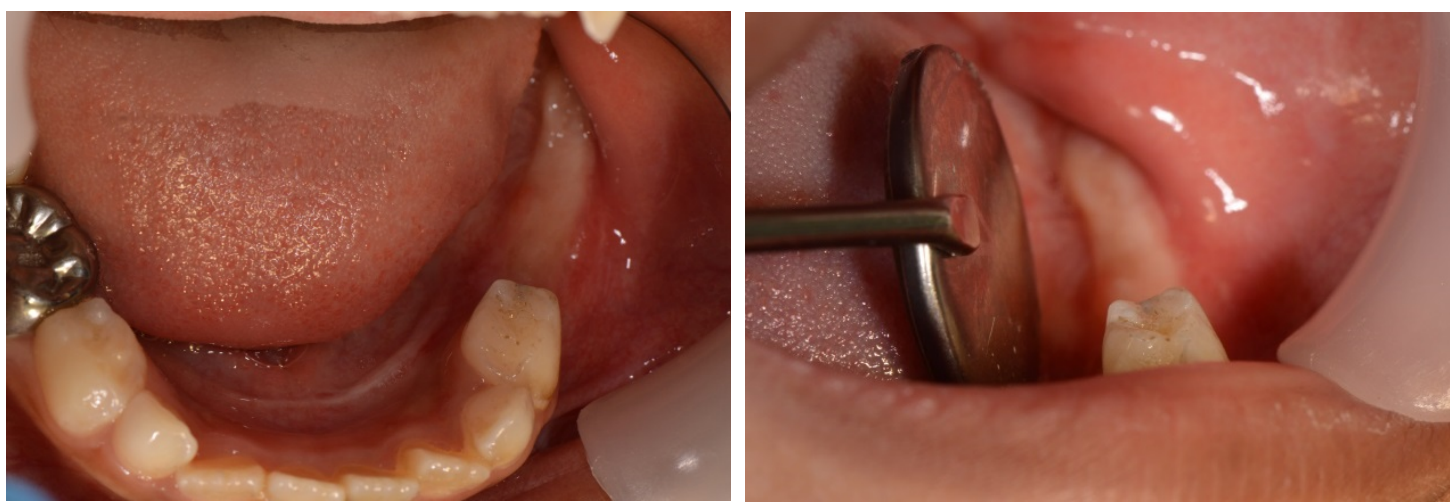

Figure 7. Intraoral photographs showing healed mandible 6 months post surgery.

Based on the histopathological and immunohistochemical findings, the diagnosis of myofibroma of left mandible was established. No evidence of recurrence was observed after 6 months follow up (Figure 7).

As it is our responsibility to ensure full mouth rehabilitation for this patient, short and long term treatment options to replace missing teeth will be explained and discussed with the parent. As an interim measure, a removable partial denture will be constructed to replace the missing lower left permanent molar until she is ready for implants. Patient will be reviewed every 6 months to monitor eruption of remaining permanent teeth and for recurrence of lesion.

\section{Discussion}

Myofibroma is a rare neoplasm that occurs due to benign proliferation of myofibroblasts. Although $90 \%$ of the lesion appear before the age of 2 , myofibroma can arise in the oral cavity at any age with no gender predilection [5]. It was first described and named by Williams and Schrum as congenital fibrosarcoma in 1951 [6]. Following a study of fibrous growth in children in 1954, Stout AP proposed the term 'congenital generalised fibromatosis' and in 1981, Chung and Ezinger used the term infantile myofibromatosis to replace Stout's term as the lesion mainly occurs in newborn and infants [3]. In 1989, Smith et al reported a solitary case of this lesion introducing the term 'myofibroma'[7]. The term myofibroma and myofibromatosis was subsequently adopted by the World Health Organisation.

The aetiology of the tumour remains unknown. Some authors have suggested autosomal dominant and recessive patterns of familial inheritance as a possibility while others have implicated trauma in the aetiopathogenesis of the lesion as injury results in myofibroblastic proliferation in the affected site $[2,4,8]$.

Myofibroma/myofibromatosis is a rare tumour presenting as solitary or multiple lesions with a predilection for soft tissues of the head and neck region. It is less common within the jaw bones.Myofibroma is thought to represent a benign proliferation of the myofibroblast, a cell with a phenotype of both fibroblast and smooth muscle cell, as demonstrated by immunohistochemical, histomorphologic and ultrastructural studies [9].

Myofibroma generally appears as a slow growing, painless and firm mass of varying sizes that may or may not be ulcerated. Due to this, patients typically appear with delayed presentation. Diversity of its clinical appearance, similarity to various benign and malignant tumours in the oral cavity in addition to being asymptomatic makes the diagnosis of myofibroma challenging. Myofibroma's clinical presentation may lead to a wide range of differential diagnosis and amongst them are pyogenic granuloma, peripheral giant cell granuloma, unicystic ameloblastoma, ameloblastic fibroma, central giant cell granuloma, odontogenic keratocyst, calcifying cystic odontogenic tumour and central odontogenic fibroma. Myofibroma can also mimic more aggressive lesions such as desmoplastic fibroma, solitary eosinophilic granuloma, osteogenic sarcoma of the mandible, fibrosarcoma and squamous cellcarcinoma. [10]. The lesion occasionally presents as a rapidly growing mass with ulceration and severe destruction of adjacent bone with ill-defined 
radiolucency on radiographs, arousing suspicion of malignancy.

Presentation of myofibroma of the mandible radiographically may range from no bony changes to unilocular or multilocular radiolucency of the mandible with sclerotic borders. Some cases may exhibit expansion and thinning of cortical plate along with displacement of associated teeth [11]. In this present case, the radiographs showed a unilocular radiolucent lesion with loss of lingual cortical plate in relation to the lower left first permanent molar. This warrants a differential diagnosis of unicystic ameloblastoma, odontogenic keratocyst, central giant cell granuloma, calcifying cystic odontogenic tumour and central odontogenic fibroma.

Histopathologically, myofibroma is a well circumscribed, unencapsulated, multinodular lesion that demonstrate biphasic pattern. The lesion comprises of two cellular components, proliferation of small, ovoid to spindle cells with scanty cytoplasm associated with numerous thinwalled branching vessels and plump spindle cells with tapering nuclei and pale cytoplasm arranged in whorled nodules and fascicles. The two components are present in variable proportions but often depict a zonal distribution. The lesion should be distinguished from other spindle cell lesions of neural, fibrohistiocytic and muscular origin. Myofibroma is negative for S100, CD34 and keratins but extensively positive for SMA. Desmin is generally negative but a focal positivity may be observed in a small subset of case.

Treatment of choice for myofibroma in the oral cavity is surgical excision to avoid recurrence. Excision of lesion would result in bleeding and a raw surgical site that might be associated with delayed healing. However, leaving the lesion in situ without any intervention will lead to discomfort and pain during speech and mastication as well as increased displacement and mobility of the associated the tooth. Hence, a dressing was placed at the surgical site, post operatively.

Recurrence of the lesion is reportedly less than $10 \%$ of cases after surgical excision [4]. These recurrences are possibly due to anatomic complexity causing difficult surgical access and incomplete excision of the lesion [12,13]. Recurrence of lesion is treated by re-excision.
Routine review of the patient would be required to assess for recurrence. We reviewed our patient on a monthly basis for the first 3 months postsurgical excision. Thereafter, patient was seen on a 3 to 6 monthly follow up. Till date, she did not have any complaints and no recurrence was reported, both clinically and radiographically.

\section{Conclusion}

Although rare, myofibroma should be considered as a differential diagnosis for solitary firm swelling in the oral cavity. Histopathological examination together with immunohistochemical analysis is essential for establishment of an accurate diagnosis.

\section{Acknowledgement}

The authors would like to thank the Director General of Health Malaysia for the permission to publish this paper.

\section{References}

[1] Vaishali N, Syed AA, Charu S, Shahela T. Myofibroma of the Gingiva: A Rare Case Report and Literature Review. Hindawi Publishing Corporation, Case Reports in Dentistry Volume 2015, Article ID 243894, 4 pages.

[2] Weiss SW, Goldblum JR (eds): Enzinger and Weiss's Soft Tissue Tumors, ed. St Louis, Mosby, 2001:357-363.

[3] Andreadis D, Epivatianos A, Samara A, Kirili T, lordanidis $F$, Poulopoulos A. Myofibroma of the Oral Mucosa: A Case Report. Med Princ Pract 2012; 21:288-291.

[4] Rubin BP, Bridge JA: Myofibroma/myofibromatosis; in Fletcher CD, Unni KK, Martene F (eds): WHO Classification of Tumors: Pathology and Genetics, Tumors of Soft Tissue and Bone. Lyon, IARC Press, 2002, pp 59-61.

[5] Zanella TA. Intraosseous Myofibroma of The Jaw-Systematic Review. Investigacao. 2015;14:182-188.

[6] Stout AP, Juvenile Fibromatosis. Cancer, 1954 Sep; 7:953-978. 
[7] Smith K.J, Skelton HG, Barrett TL, Lupton GP, Graham JH. Cutaneous Myofibroma. Modern Patholog.1989 Nov; 2(6) : 603-609.

[8] Darby I, Skalli O, Gabbiani G. Alpha-smooth muscle actin is transiently expressed by myofibroblasts during experimental wound healing. Lab. Investig.1990; 63:21-29.

[9] McMenamin ME, Fletcher CD. Malignant myopericytoma: Expanding the spectrum of tumours with myopericytic differentiation. Histopathology. 2002;41:450-60.

[10] Birendra Rai, Evodia Ludusan, Brianan McGovern, Farhana Sharif. Mandibular swelling in a 5-year-old child - mandibular myofibroma. BMJ Case Rep 2014; 2014: bcr2014203977.

[11] Venkatesh V, Batchu PV, Jeevan Kumar KA, Mohan AP. Myofibroma - A rare entity with unique clinical presentation. J Maxillofac Oral Surg. 2015 Mar: 14(Suppl 1): 64-68.

[12] Beck JC, Devaney KO, Weatherly RA, Koopmann Jr CF, Lesperance MM. Pediatric myofibromatosis of the head and neck. Arch Otolaryngol Head Neck Surg 1999 Jan:125:39-44.

[13] Anita D, Karla C, Poonam S, Anita S, Shaheen S. Solitary Intra Osseous Myofibroma of the Jaw: A Case Report and Review of the Literature. Children (Basel). 20170ct; 4(10):91. 